\title{
Preface
}

\section{Sleep Hypoventilation: A \\ State-of-the-Art Overview}

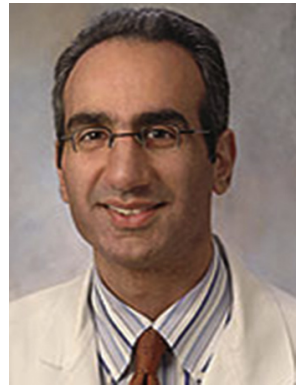

Babak Mokhlesi, MD, MSc

Editor
The association between obesity and hypoventilation has long been recognized. Of historical interest, obesity hypoventilation syndrome was described well before obstructive sleep apnea was recognized in 1969. Since then, we have made great strides to advance our understanding of sleep hypoventilation syndromes. Sleep hypoventilation, particularly obesity hypoventilation syndrome, should no longer be considered a rare condition in the current global epidemic of obesity. Despite the increased recognition of this condition by health care providers, a significant proportion of the population afflicted by sleep hypoventilation remains undiagnosed and untreated, and the potential societal, health-related, and economic consequences remain poorly defined. It therefore gives me great pleasure to see the outstanding contributions from a cast of highly qualified experts and researchers who discuss in great detail the recent advances in epidemiology, pathophysiology, diagnosis, and treatment of adult and pediatric sleep hypoventilation syndromes.

While extensive research spanning several decades has increased our understanding of sleep hypoventilation syndromes, the specific purpose of this issue of Sleep Medicine Clinics is to provide a forum that allows a group of internationally recognized experts in the field to thoroughly and critically review the current state of knowledge and to provide insight and guidance on important questions that require further investigation. My most fervent hope is that the state-of-the-art discussions in this issue will stimulate the readership and provide a focused assessment of the current state of knowledge on sleep hypoventilation. I am confident that the outstanding contributions by my colleagues will help current and future investigators and clinicians to formulate many probing questions waiting to be asked and help us find the answers in this exciting and important area of respiratory and sleep medicine.

The issue begins with an article providing a historical perspective on sleep hypoventilation syndromes. The following article provides an overview of the pathophysiology of sleep hypoventilation and how it can progress from hypoventilation during sleep to hypoventilation during wakefulness. This is followed by an in-depth discussion regarding diagnostic considerations and technological limitations related to capnometry. The next article provides an update of advances in various modalities of positive airway pressure therapy. Scoring respiratory events during noninvasive ventilation can be challenging, and a systematic description of these events has been summarized in the following article. The next three articles provide a detailed and up-to-date summary of diagnosis, epidemiology, and treatment of obesity hypoventilation syndrome. These three articles are followed by a series of outstanding reviews covering the spectrum of hypoventilation, 
such as the overlap syndrome, neuromuscular disorders, hypoventilation due to opioids and sedatives, as well as the interaction between anesthesia and sleep hypoventilation syndromes. The last two articles provide a comprehensive review of pediatric hypoventilation syndrome and treatment with noninvasive ventilation in children.

I am indebted to my colleagues who graciously accepted my invitation to make exceptional contributions to this issue of Sleep Medicine Clinics. I am also grateful to Dr Teofilo Lee-Chiong for providing a forum that allows an in-depth analysis and discussion of the topic. I hope you will enjoy reading these outstanding articles.

Babak Mokhlesi, MD, MSc Section of Pulmonary and Critical Care Sleep Disorders Center and Sleep Fellowship

Program

University of Chicago

5841 S. Maryland Avenue MC6076/Room M630

Chicago, IL 60637, USA

E-mail address:

bmokhles@medicine.bsd.uchicago.edu 